\title{
Formant Frequencies under Cognitive Load: Effects and Classification
}

\author{
Tet Fei Yap, ${ }^{1,2}$ Julien Epps, ${ }^{1,2}$ Eliathamby Ambikairajah,, 2 \\ and Eric H. C. Choi (EURASIP Member) ${ }^{2}$ \\ ${ }^{1}$ School of Electrical Engineering and Telecommunications, The University of New South Wales, \\ Sydney NSW 2052, Australia \\ ${ }^{2}$ ATP Research Laboratory, National ICT Australia (NICTA), Eveleigh NSW 2015, Australia
}

Correspondence should be addressed to Tet Fei Yap, tetfei.yap@nicta.com.au

Received 1 August 2010; Accepted 20 December 2010

Academic Editor: Shrikanth Narayanan

Copyright (C) 2011 Tet Fei Yap et al. This is an open access article distributed under the Creative Commons Attribution License, which permits unrestricted use, distribution, and reproduction in any medium, provided the original work is properly cited.

\begin{abstract}
Cognitive load measurement systems measure the mental demand experienced by human while performing a cognitive task, which is useful in monitoring and enhancing task performance. Various speech-based systems have been proposed for cognitive load classification, but the effect of cognitive load on the speech production system is still not well understood. In this work, we study formant frequencies under different load conditions and utilize formant frequency-based features for automatic cognitive load classification. We find that the slope, dispersion, and duration of vowel formant trajectories exhibit changes under different load conditions; slope and duration are found to be useful features in vowel-based classification. Additionally, 2-class and 3-class utterance-based classification results, evaluated on two different databases, show that the performance of frame-based formant features was comparable, if not better than, baseline MFCC features.
\end{abstract}

\section{Introduction}

Cognitive load refers to the load imposed by a certain task on the cognitive system of a person [1]. Cognitive load theory, first developed in the field of educational psychology, was originally focused on improving the process of acquiring and applying new knowledge [2]. Central to cognitive load theory is the notion that working memory is required while performing a cognitive task, but this working memory is limited. As a cognitive task becomes more challenging, the amount of working memory required to complete the task will typically increase. When the working memory requirement exceeds the available capacity, task performance will deteriorate.

In recent years, there has been a growing interest in monitoring and measuring cognitive load as a means to monitor or even enhance human task performance. Applications expected to benefit from such cognitive load monitoring systems include air traffic control systems [3], in-car user interfaces [4] and military human-machine systems [5].

There are many different methods available for cognitive load measurement, such as pupil dilation and heart rate variability [1]. Nevertheless, speech-based methods are attractive because they are nonintrusive and widely available and can potentially be real-time. Various speech parameters have been correlated with cognitive load during early investigations of the problem [6]. Sentence fragments, articulation rate [7], and filled pauses [8] have also been proposed as features for cognitive load measurement. However, most of these proposed features are high level features and the cognitive load classification methods proposed were not fully automatic. In 2008, Yin et al. proposed the use of a Gaussian mixture model- (GMM-) based classifier to automatically classify different cognitive load levels [9]. In this system, the front-end features used were frame-based acoustic features: mel-frequency cepstral coefficients (MFCCs), pitch, and intensity. MFCCs are a set of features commonly used in various speech processing applications, and they capture information in the magnitude part of the speech spectrum. Pitch and intensity, on the other hand, are features that capture information relating to the prosody of speech.

In recent years, various frame-based features have been proposed to improve on the previously proposed baseline 
system $[10,11]$, and MFCCs seem to have emerged as an effective set of baseline features for cognitive load classification. However, MFCCs do not provide us with any insight into how cognitive load affects the speech spectrum or the underlying speech production system. Moreover, MFCCs may have higher dimensionality than what is strictly required for the problem.

Previously, glottal features were investigated in an attempt to link cognitive load to the speech production system $[11,12]$. A natural extension to these pieces of work would be to study the effect of cognitive load on the vocal tract. In this respect, an investigation of formant frequencies for cognitive load classification is interesting and complementary. This is because formant frequencies (the frequencies at which broad spectral peaks occur in the magnitude spectrum of speech) are closely related to the underlying configuration of the vocal tract.

Lively et al. have previously found no significant differences in the first three formant frequencies (F1, F2, F3) under different workload conditions. However, more recent investigations have shown that formant frequencies do carry information useful for cognitive load classification $[13,14]$. Moreover, formants have also been investigated in two other fields, which we believe are related to cognitive load classification: stress classification [15] and emotion recognition $[16,17]$.

In the field of emotion recognition, statistics calculated from the formant frequencies have been used successfully as part of a larger feature set for emotion recognition [18]. It was argued that using the statistics of low-level descriptors of speech is beneficial, because the reduction in information helps avoid phonetic overmodeling [18].

In this work, we analyze statistical parameters of different vowel formant frequencies, such as standard deviation, minima, and skewness, in order to better understand the effect of cognitive load on the formant trajectory. This is an extension of our previous work, which focused only on the analysis of the vowel formant means [14]. Besides that, we also analyze the formant trajectories in greater detail: in terms of the trajectory in the F1-F2 plane, and also in terms of the time-normalized trajectory for a particular speaker.

Although the performance of frame-based formant features for cognitive load classification has been reported in our previous work $[13,14]$, classification was performed on a per-utterance basis on a single database. In this work, the performance of frame-based formant features is verified on a second database, and a system employing statistical formant features extracted on a per-vowel basis is proposed.

\section{Cognitive Load Databases}

2.1. Stroop Test Database. The Stroop test database, reported previously [9], consists of 16 randomly selected native English speakers ( 7 males and 9 females) performing three tasks of varying cognitive load levels. In the low-load task, the speakers were required to read aloud words corresponding to different color names. In the medium-load task, there was a mismatch between the color names and the font colors, and the speakers were asked to name the font colors instead.
The high-load task was similar to the medium-load task except that time constraints were introduced to the task.

Each task produced an utterance with average duration of 16 seconds (duration of effective speech is 7 seconds). In each utterance, 20 color names were spoken: 10 different colors randomly repeated twice. The color names that were used are "black", "blue”, "brown", "gray", "green”, "orange”, "pink”, "purple", "red", and "yellow".

Database recording was conducted in two separate sessions. Each speaker was required to record 2 utterances per load level in each session. However, a small number of speakers were unable to attend both recording sessions. Hence, for each speaker, a maximum of 4 utterances were recorded per load.

In total, 161 utterances were recorded, and approximately 54 utterances were obtained per load level.

2.2. Reading-Comprehension Database. This database consists of 15 randomly selected native English speakers ( 7 males and 8 females) reading aloud 3 text passages of varying difficulty levels, and answering open-ended questions related to the passages that they have read [19]. The difficulty level of the text was measured using the Lexile Framework for Reading [20], which measures the semantic difficulty and syntactic complexity of a particular text. It ranges from $200 \mathrm{~L}$ (Lexiles) for beginner readers to above $1700 \mathrm{~L}$ for advanced text. Lexile ratings of the text selected for the low, medium, and high-load cases were $925 \mathrm{~L}, 1200 \mathrm{~L}$, and $1350 \mathrm{~L}$, respectively.

After the text passage reading, participants were asked to answer three open-ended questions.

(i) Give a short summary of the story in at least five whole sentences.

(ii) What was the most interesting point in this story?

(iii) Describe at least two other points highlighted in this story.

For the high-load task, participants were asked to perform an additional dual task. Throughout the task, random 2 digit numbers were played softly in the background (via a headset) at random intervals. The participants were asked to count how many numbers were heard throughout the reading and comprehension task.

In this work, only the utterances from the comprehension tasks were used. A total of 135 utterances were recorded, that what is, 45 utterances per load level. Each utterance corresponded to the answer to one of the three open-ended questions in the comprehension task. The average duration of each utterance is approximately $32 \mathrm{~s}$ (duration of effective speech is $12 \mathrm{~s}$ ).

2.3. Story Reading Database. Speakers who participated in the Stroop test database collection also recorded a separate story reading task. The average duration of the recordings from each speaker is approximately $90 \mathrm{~s}$ (duration of effective speech is $38 \mathrm{~s}$ ). Speech from this database is herein used for background model training purposes. 


\section{Effect of Cognitive Load on Vowel Formants}

3.1. Experimental Setup. The experiments detailed in this section are aimed at investigating the changes in the formant trajectories under different cognitive load conditions. The experiments were performed on a subset of the Stroop test database, and a total of 5 vowel sounds were considered: /eh/, /iy/, /ae/, /uw/, and /ao/ extracted from the color words "red", "green", "black", "blue", and "orange", respectively.

Only vowels spoken under the low and medium cognitive load conditions were considered here. This is because the high-load task design induces an increase in rate of speech. This change in speech rate confounds our analysis, given that speech rate has been shown to affect formant frequencies [21, 22]. For example, we observed a smaller vowel plane area for high cognitive load conditions, compared with medium-load conditions. This reduction in vowel plane area is expected when speech rate is increased [21]; thus, it is not sure if cognitive load is also accountable for this effect. Hence, we focused our analyses in this section on low and medium cognitive load tasks, wherein changes in speech rate were not explicitly induced.

To obtain the vowel formants, the color words were first manually segmented from the full utterances in the Stroop test database. The vowel boundaries were then determined by performing forced phone alignment on the color words, using the HVite program in the Hidden Markov Model Toolkit (HTK). The phone models were trained, using MFCC features, on the training partition of the TIMIT corpus [23]. When tested on the test partition of the TIMIT corpus, the phone alignment system achieved an accuracy rate of $87.2 \%$ with a temporal tolerance of within 20 milliseconds.

The first three formant frequencies were then extracted from each vowel sound using the Wavesurfer/Snack toolkit [24]. A 49 milliseconds Hamming window was applied with a frame increment of $10 \mathrm{~ms}$. Otherwise, all other parameters remained at the default settings: the number of formants tracked (four), preemphasis factor (0.7), LPC order (12), nominal value of first formant frequency $(500 \mathrm{~Hz})$, and sampling frequency $(10 \mathrm{kHz})$. Erroneous formant values were manually corrected using a spectrogram.

\subsection{Vowel Formant Statistical Parameters}

3.2.1. Method. Formant parameters that were used in this study include the following.

(1) First is the statistical parameters such as mean (Mean), standard deviation (StdDev), maximum (Max), minimum (Min), range (Range), skewness (Skewness), and kurtosis (Kurtosis) of the vowel trajectory.

(2) Second is the formant values and slopes at different equidistant temporal positions. Formant20, Formant50, and Formant80 represent the formant values at the $20 \%, 50 \%$, and $80 \%$ points on the vowel trajectory, whereas Slope20 and Slope 80 represent the local slope at the $20 \%$ and $80 \%$ points on the vowel trajectory.
(3) Third is the DCT coefficients that model the entire vowel formant trajectories. For the vowel formant trajectory $x(n)$ with $N$ total number of frames, these were calculated as follows:

$\mathrm{DCT}_{k}=w(k) \sum_{n=1}^{N} x(n) \cos \left(\frac{\pi(2 n-1)(k-1)}{2 N}\right)$,

where $k=1,2, \ldots, N$ and

$$
w(k)= \begin{cases}\frac{1}{\sqrt{N}}, & k=1, \\ \sqrt{\frac{2}{N}}, & 2 \leq k \leq N .\end{cases}
$$

(4) Fourth is the regression coefficients for linear and quadratic curves. LinRegSlope and LinRegIntercept represent the slope and intercept of the linear regression line that best fits the vowel formant trajectory, whereas QuadRegOrd0, QuadRegOrd1, and QuadRegOrd2 represent the first 3 coefficients of the quadratic regression curve that best fits the trajectory.

(5) Fifth is the total number of frames for the formant trajectory (Duration). The trends of Duration, observed for F1, F2, and F3, should all be identical since the three formants should have the same duration for the same vowel sound.

The above parameters were calculated separately for each formant frequency, vowel sound, and load level. The parameters were then averaged for each speaker and each session. $T$-tests were then performed (for each color and each formant) on the differences of the means of the parameters between low and medium-load. Approximately 27 test samples were used for each test.

3.2.2. Results and Discussion. Figure 1 shows the mean F1 and F2 values, averaged across all speakers and sessions, for different vowels and different cognitive load conditions. The figure suggests that the mean formant values do shift for different load conditions, and this shift is vowel-dependent. However, we did not observe any shrinking of the vowel plane that might have suggested vowel reduction; this agrees with the observations reported by Lively et al. [6].

Table 1 summarizes the significant trends $(P<.1, P<$ $.05, P<.01)$ for various formant parameters as cognitive load level changes. The direction of the arrows indicates the trends as cognitive load increases from low to medium. Due to the small sample size, only strong trends showed significance. The following is a discussion of the observed trends.

Mean Value and DC Offset. Table 1 shows that only the mean F2 values in /ao/ exhibit a significant decreasing trend. This can be compared with the results of Lively et al. [6], which found no statistical differences in formant frequencies under different cognitive workload conditions. However, in another work, Hansen and Patil [15] reported that the first 3 formant frequencies increase when speech is spoken under stress. 
TABle 1: Trends of different formant parameters as cognitive load increases from low to medium. Parameters are calculated from the individual formant trajectory of particular vowel sounds. One, two, and three arrows indicate that the trend is significant for $P<.1, P<.05$, and $P<.01$, respectively.

\begin{tabular}{|c|c|c|c|c|c|c|c|c|c|c|c|c|c|c|c|}
\hline \multirow[t]{2}{*}{ Feature } & \multicolumn{5}{|c|}{ F1 } & \multicolumn{5}{|c|}{ F2 } & \multicolumn{5}{|c|}{ F3 } \\
\hline & /eh/ & /iy/ & /ae/ & /uw/ & /ao/ & /eh/ & /iy/ & /ae/ & /uw/ & /ao/ & /eh/ & /iy/ & /ae/ & /uw/ & /ao/ \\
\hline Mean & & & & & & & & & & $\downarrow$ & & & & & \\
\hline LinRegIntercept & & & & & $\downarrow$ & & & $\downarrow$ & & $\downarrow$ & & & & & \\
\hline QuadRegOrdo & & & & & $\downarrow \downarrow$ & & & $\downarrow \downarrow$ & & $\downarrow \downarrow$ & & & & & \\
\hline$\overline{D C T_{1}}$ & $\uparrow \uparrow \uparrow$ & $\uparrow \uparrow$ & $\uparrow \uparrow \uparrow$ & $\uparrow \uparrow \uparrow$ & & $\uparrow \uparrow \uparrow$ & $\uparrow \uparrow$ & $\uparrow \uparrow$ & $\uparrow \uparrow \uparrow$ & & $\uparrow \uparrow \uparrow$ & $\uparrow \uparrow$ & $\uparrow \uparrow$ & 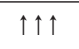 & $\uparrow$ \\
\hline Duration & $\uparrow \uparrow \uparrow$ & $\uparrow \uparrow$ & $\uparrow \uparrow$ & $\uparrow \uparrow \uparrow$ & $\uparrow \uparrow$ & $\uparrow \uparrow \uparrow$ & $\uparrow \uparrow$ & $\uparrow \uparrow$ & $\uparrow \uparrow \uparrow$ & $\uparrow \uparrow$ & $\uparrow \uparrow \uparrow$ & $\uparrow \uparrow$ & $\uparrow \uparrow$ & $\uparrow \uparrow \uparrow$ & $\uparrow \uparrow$ \\
\hline StdDev & & & & $\uparrow \uparrow \uparrow$ & & & $\uparrow$ & $\uparrow$ & & & & & & & $\uparrow \uparrow \uparrow$ \\
\hline Min & & & & & & & & $\downarrow \downarrow$ & & $\downarrow \downarrow$ & & & & & $\downarrow \downarrow$ \\
\hline $\operatorname{Max}$ & & & & $\uparrow \uparrow$ & & & $\uparrow$ & & & & & & & & \\
\hline Range & & & & $\uparrow \uparrow \uparrow$ & & & & $\uparrow \uparrow$ & & & & & & & $\uparrow \uparrow \uparrow$ \\
\hline Formant20 & & & & & & & & $\downarrow$ & & $\downarrow \downarrow$ & & & & & \\
\hline Formant80 & & & & & & & & & $\downarrow$ & & & & & & \\
\hline Formant50 & & & & & & & & & & & & & & & \\
\hline Slope20 & & & & $\downarrow \downarrow$ & & & & & & & & & & & \\
\hline Slope80 & & & & & & & & & & & & & & & \\
\hline LinRegSlope & & & & $\uparrow \uparrow$ & & & & & $\downarrow \downarrow$ & $\uparrow$ & $\downarrow$ & & & & \\
\hline $\mathrm{DCT}_{2}$ & & & & $\uparrow \uparrow$ & & & & $\downarrow \downarrow$ & & $\downarrow$ & & & & & $\uparrow \uparrow \uparrow$ \\
\hline $\mathrm{DCT}_{3}$ & & & & & & & $\downarrow$ & & & & & & & $\uparrow$ & \\
\hline Skewness & & $\uparrow$ & & & & & $\uparrow$ & & & & $\downarrow \downarrow$ & & & & \\
\hline Kurtosis & $\uparrow$ & & & & & & $\downarrow \downarrow$ & & & & & & & & \\
\hline QuadRegOrd1 & & & & & & $\downarrow$ & & & & & & & & & \\
\hline QuadRegOrd2 & & & & & & $\uparrow \uparrow \uparrow$ & & & & & & & & & \\
\hline
\end{tabular}

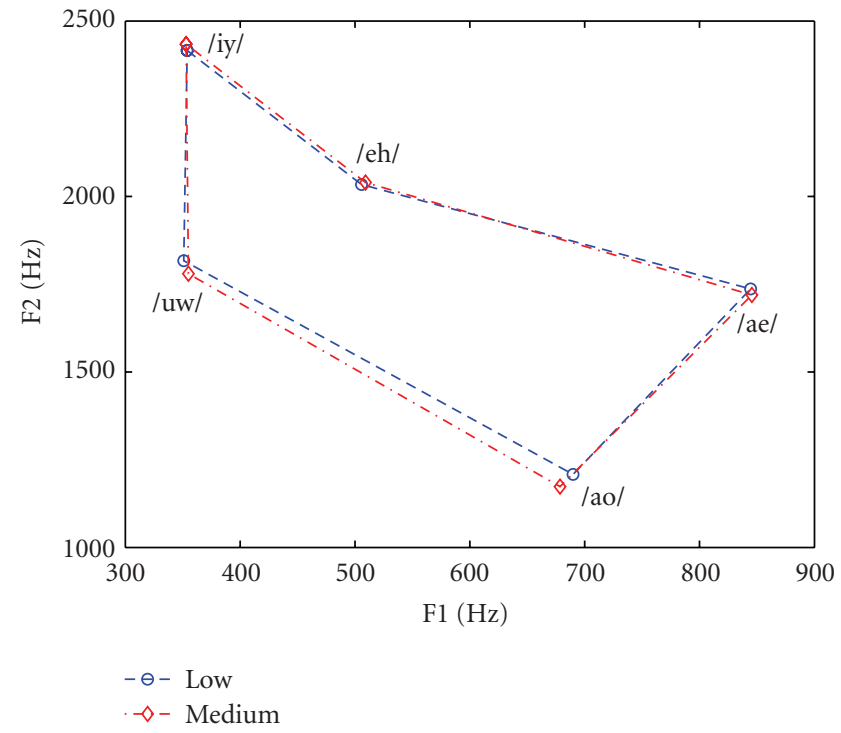

Figure 1: Mean F1 and F2 for different vowel sounds, spoken under low- and medium-load conditions, averaged across all speakers and sessions (after [14]).

Apart from the database size, we believe that the lack of significance in the mean vowel formants may be due to errors in calculating the vowel boundary. As the vowels were extracted from color words, the vowel boundaries were less clearly defined as compared to, say, an hVd word. Errors in determining the vowel boundaries, hence, caused neighboring phonemes to affect the mean values of the vowel formants.

The linear and quadratic regression intercepts (LinRegIntercept and QuadRegOrd0) are related to the DC offset of the trajectories, and they are less affected by the previously mentioned problem. Here, the results show that the two parameters exhibit decreasing trends, in F1 for /ao/ and F2 for $/ \mathrm{ae} /$ and $/ \mathrm{ao} /$, as cognitive load increases.

First DCT Coefficient and Duration. The highly significant increasing trends observed for the first DCT coefficient $\left(\mathrm{DCT}_{1}\right)$, at first glance, seem to contradict the results for the mean vowel formants; the difference lies in the scaling factor $1 / \sqrt{N}$ present in the DCT calculation ((1) and (2)). Compared with the equation for mean calculation over the same interval, $\mathrm{DCT}_{1}$ is actually the mean weighted by $\sqrt{N}$. Hence, the strong significant differences in the $\mathrm{DCT}_{1}$ values across different load levels should be considered to be dominated by duration effects.

When a $t$-test was performed on Duration for low and medium-loads, a consistent increase was observed for all vowel sounds. Hence, vowel duration increases as cognitive load increases; that is, subjects tend to speak more slowly for medium cognitive load, compared with low load. 
Within-Trajectory Dispersion. The standard deviation of the formant trajectories (StdDev) shows an increasing trend, as cognitive load increases, in F1 for /uw/, F2 for /iy/ and /ae/, and F3 for /ao/. This is consistent with the increasing trend observed for the trajectory range (Range). As expected from the increasing StdDev, the trajectory minimum (Min) exhibits a decreasing trend (in F2 for /ae/ and /ao/), whereas the trajectory maximum (Max) exhibits an increasing trend (in F1 for /uw/ and F2 for /iy/).

Time-Normalized Formant Parameters. The formant frequencies at the $20 \%, 50 \%$, and $80 \%$ points in the timenormalized trajectory (Formant20, Formant50, Formant80) do not show much significant differences between cognitive load levels. This might, again, be due to errors in vowel boundary calculations causing formant frequencies to be calculated at the wrong temporal points. Similarly, few significant differences can be observed for the formant trajectory slope at the $20 \%$ and $80 \%$ points in the timenormalized trajectory (Slope20, Slope80).

Slope-Based Formant Parameters. In contrast to Slope20 and Slope80, the linear regression slope of the formant trajectory (LinRegSlope) shows an increasing trend, in F1 for /uw/ and $\mathrm{F} 2$ for /ao/, and a decreasing trend, in F2 for / uw/ and F3 for /eh/. The second DCT coefficient $\left(\mathrm{DCT}_{2}\right)$ also displays significant trends, in F1 for /uw/, in F2 for /ae/ and /ao/, and in F3 for /ao/. These results indicate that cognitive load does affect the direction of the formant trajectory.

In general, the results suggest that the DC offset, dispersion, and the slope of the formant trajectory carry cognitive load-related information, at least for certain vowel sounds. Furthermore, vowel duration seems to be strongly affected by cognitive load.

3.3. Vowel Formant Trajectories in the F1-F2 Plane. Analysis in the previous section focused on individual formant frequencies of different vowel sounds. However, it is common to analyze the vowel formant frequency trajectories from the perspective of the F1-F2 plane.

Figure 2 shows the average time-normalized formant trajectories for different vowel sounds and different load levels, averaged across all speakers and sessions. The arrows indicate the directions in which the trajectories are moving with time. The time-normalized formant trajectory was obtained by using linear interpolation to calculate the formant values at 11 equidistant temporal locations.

To quantify the changes in the formant trajectory, we calculated the formant trajectory length TL. This parameter is basically a piecewise approximation of the formant trajectory length in the F1-F2 plane. It provides a simple measure to determine the magnitude of the formant trajectory change occurring in the F1-F2 plane. The calculation of TL is based on the equation given by Fox and Jacewicz [25]:

$$
\mathrm{TL}=\sum_{k=1}^{K} \sqrt{\left(F 1_{k}-F 1_{k+1}\right)^{2}+\left(F 2_{k}-F 2_{k+1}\right)^{2}},
$$

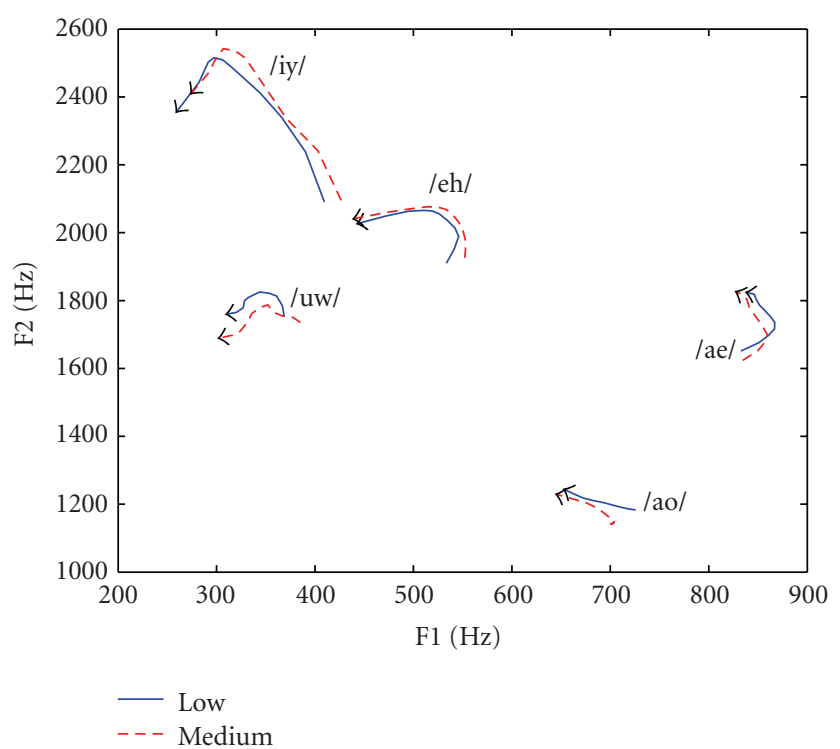

FIGURE 2: Time-normalized formant trajectories for different vowel sounds, spoken under low, and medium-load, averaged across all speakers and sessions (after [14]).

TABLE 2: Trajectory length TL of time-normalized formant trajectory, averaged across all speakers and sessions, for different vowel sounds and load conditions.

\begin{tabular}{lcc}
\hline Vowel sound & $\begin{array}{c}\text { Low-load } \\
(\mathrm{Hz})\end{array}$ & $\begin{array}{c}\text { Medium-load } \\
(\mathrm{Hz})\end{array}$ \\
\hline /eh/ & 249 & 249 \\
/iy/ & 606 & 603 \\
/ae/ & 188 & 211 \\
/uw/ & 159 & 179 \\
/ao/ & 95 & 117 \\
\hline
\end{tabular}

where $F 1_{k}$ and $F 2_{k}$ are the $\mathrm{F} 1$ and $\mathrm{F} 2$ values at the $k$ th equidistant location of the vowel formant trajectory. $K+1$ is the total number of equidistant points to consider. $K=10$ was chosen for this experiment.

Table 2 summarizes the value of $T L$ for different vowel sounds spoken under different load conditions. TL for the vowel sounds /eh/ and /iy/ remains roughly unchanged as cognitive load increases. Looking at the formant trajectory in Figure 2, we can see that the trajectory shapes for the two vowel sounds remain roughly the same. This implies that any changes, due to cognitive load, for the two vowel sounds are reflected as shifts in the mean values of the formant trajectory.

On the other hand, TL for the vowel sounds /ae/, /uw/, and /ao/ seems to be higher for medium-load conditions. This suggests that there are more changes in the shape of the trajectory for those vowel sounds; the differences in the trajectory shape can be observed in Figure 2. This finding is consistent with the results reported in Table 1, wherein significant differences are mostly observed in the /ae/, /uw/ and /ao/ sounds. This raises the prospect that certain vowel 


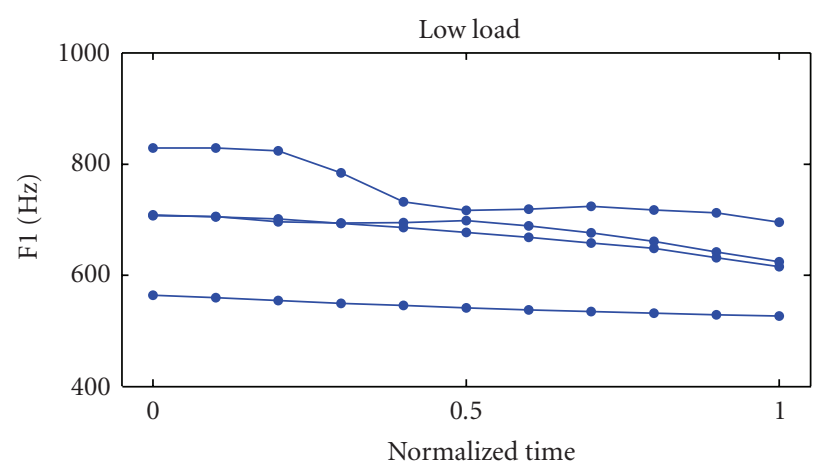

(a)

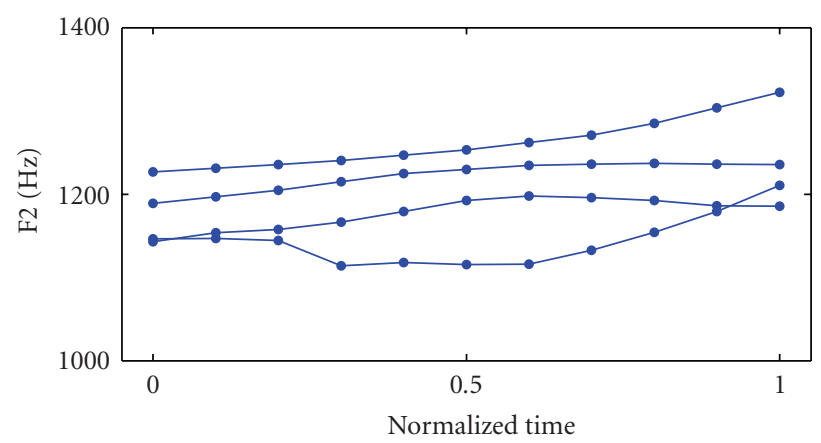

(c)

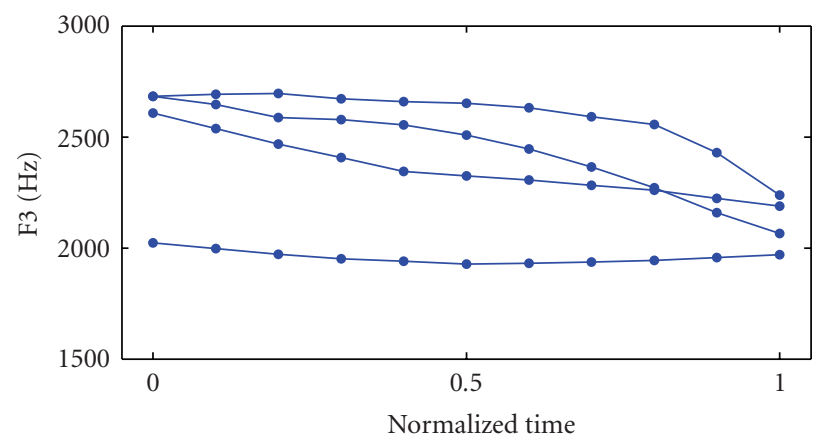

(e)

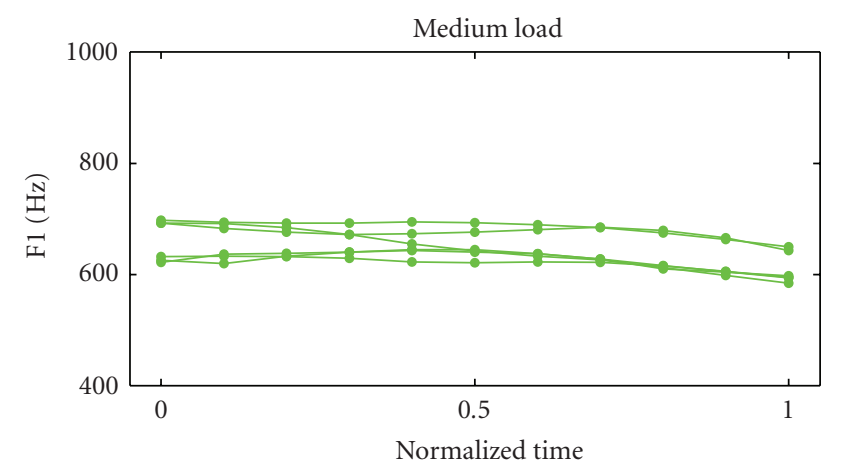

(b)

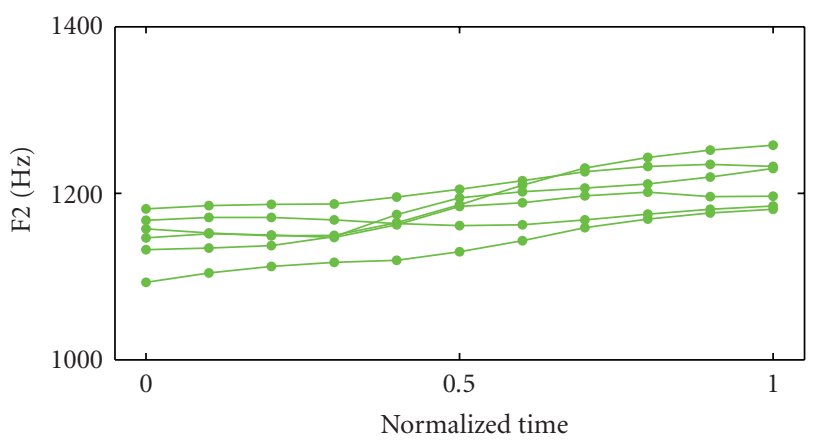

(d)

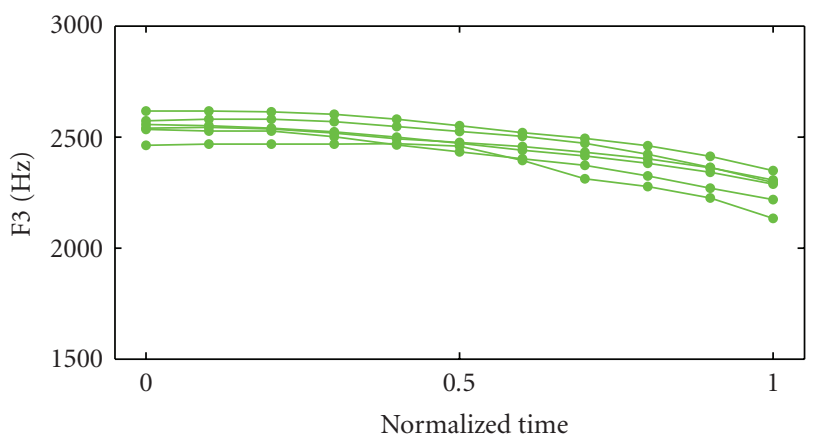

(f)

FIGURE 3: Time-normalized F1, F2, and F3 trajectory for the vowel /ao/, spoken by a particular speaker at a particular session, under lowand medium-load conditions.

sounds might contain more useful information for cognitive load discrimination.

3.4. Vowel Formant Trajectories of an Individual Speaker and Session. The analyses performed in the previous sections have focused on the mean values of different formant parameters averaged across all speakers and sessions. However, it is also useful to look at the vowel formant trajectory of a particular speaker at a particular session, in addition to aggregate data.

Figure 3 shows the time-normalized first 3 formant trajectories of the vowel /ao/, under low- and medium-load conditions, uttered by a particular speaker at a particular session. This speaker/session was selected to represent typical trajectories found in the database. From this figure, it seems that the variance of formant trajectories between different vowel instances tends to decrease when cognitive load changes from low to medium. This effect can be observed for all three formant trajectories.

Other observations with respect to Figure 3 can be related to Table 1, and they seem to agree with each other. For example, the figure indicates a decreasing mean F1 and F2 trajectory, as cognitive load increases; the decrease in F2 agrees with the decreasing trend of the mean formants reported in Table 1. Additionally, the figure also shows that formant value shifts, due to cognitive load changes, seem to be more prominent at the vowel onset region, especially for the case of F2. This observation, again, agrees with the significant decreasing trend of Formant20 in F2 for /ao/; Formant20 represents the formant values at the $20 \%$ point (vowel onset region).

In summary, although not all findings in this section may generalize to other speakers in the database, the 
results provide tangible examples of the findings reported in Section 3.2, within a single speaker and session.

\section{Cognitive Load Classification Using Formant Features}

4.1. Vowel-Based Classification Using Statistical Formant Features. In this section, we seek to determine if the formant parameters that we studied in Section 3.2 can be used as features for vowel-based cognitive load classification. This experiment was conducted on the Stroop test database.

4.1.1. Experimental Setup. The method used to extract the vowel formants was similar to the method described in Section 3.1. The only differences here are that the formant frequencies were not manually corrected, and all vowel sounds from the database were used. These included /ae/ from the word "black", /uw/ from "blue", /aw/ from "brown", /ey/ from "gray", /iy/ from "green", /ao/, /ah/, and /ih/ from "orange", /ih/ from "pink", /er/, /el/, and /ah/ from "purple", /eh/ from "red", and /eh/ and /ow/ from "yellow".

Speaker-specific feature warping $[13,26]$ was applied to the formant frequencies to remove interspeaker variability of the formant frequencies; one source of variability is the differences in the vocal tract lengths among speakers. Speaker-specific feature warping seeks to map the probability distribution of the pooled formant frequencies of each speaker to the standard normal distribution. It is applied to each feature dimension separately. For a given formant value $p$ for speaker $S$, the warped feature value $q$ is given by the equation $q=H^{-1}((N+(1 / 2)-R) / N)$, where $H^{-1}()$ denotes the normal inverse cumulative distribution function, $N$ is the total number of formant values for speaker $S$, and $R$ is the ranking of $p$ after sorting the formant values for speaker $S$ in descending order. A detailed explanation can be found in [26].

For each normalized formant trajectory of a particular vowel sound, different statistical parameters were extracted: Mean, LinRegIntercept, QuadRegOrd0, DCT 1 , Duration, StdDev, Min, Range, LinRegSlope, $D C T_{2}$, and Skewness. These formant parameters are a subset of the parameters analyzed in Section 3.2. Apart from Mean, these parameters were chosen because they exhibited statistically significant differences between low and medium cognitive load for at least 3 cases (across different formant frequencies and vowel sounds).

A GMM classifier with universal background model (UBM) training was then used to classify the vowel sounds in a leave-one-speaker-out cross-validation evaluation. The number of frames available for training was limited, since multiple frames of a vowel trajectory produced only one feature value per feature dimension. Hence, a UBM with a low number of Gaussian mixtures (8 Gaussians) was trained, using the vowel sounds from the entire database. The means of the UBM were then adapted with maximum a posteriori (MAP) adaptation, using vowel sounds that corresponded to a particular load level.

During classification, the likelihood scores for all vowels belonging to the same utterance were averaged, and the class decisions were made for each utterance instead of each
TABLE 3: 2-class and 3-class accuracy results of vowel-based classification using different statistical formant features.

\begin{tabular}{lccc}
\hline Features & Dimension & $\begin{array}{c}\text { 2-class accuracy } \\
(\%)\end{array}$ & $\begin{array}{c}\text { 3-class accuracy } \\
(\%)\end{array}$ \\
\hline Mean & 3 & 45.4 & 37.3 \\
LinRegIntercept & 3 & 51.9 & 41.0 \\
QuadRegOrd0 & 3 & 52.8 & 40.4 \\
DCT $_{1}$ & 3 & 67.6 & 49.0 \\
Duration & 1 & 71.3 & 45.8 \\
StdDev & 3 & 55.6 & 37.2 \\
Min & 3 & 63.9 & 50.9 \\
Range & 3 & 63.0 & 46.6 \\
LinRegSlope & 3 & 69.4 & 44.6 \\
DCT 2 & 3 & 63.9 & 39.7 \\
Skewness & 3 & 47.2 & 33.5 \\
\hline
\end{tabular}

vowel. This setup reflects a real-life system better, whereby a decision is made not based on a single vowel sound, but on a longer speech utterance.

Unlike Section 3 where the high-load task was not considered in the analysis, the classification experiments here were performed both with and without the high-load task. This is because the high-load task (with the explicit change in speech rate) represents, to some extent, data in real-world situations. Hence, including the high-load task allowed us to evaluate the effectiveness of the classification system in realworld situations.

4.1.2. Results and Discussion. Table 3 shows the 2-class (low and medium-load) and 3-class (low, medium, and highload) classification performance of a vowel-based cognitive load classification system, using different statistical formant features. The features have 3 dimensions, each dimension corresponding to a formant; Duration has only one dimension since the durations for the first three formant frequencies are the same.

The 2-class and 3-class results reported in Table 3 seem to agree with the statistical analysis found in Table 1: statistical parameters that show more significant differences in Table 1 produce better classification accuracies when applied as features in the vowel-based cognitive load classification.

Results show that reasonably good performance was observed for parameters related to the formant trajectory slope (LinRegSlope and $D C T_{2}$ ), trajectory range (Min and Range), and vowel length (Duration, $D C T_{1}$ ). 2-class results show that the best performing features are Duration and LinRegSlope, whereas the 3-class results show that the best performing feature is Min followed by $D C T_{1}$.

Mean, on the other hand, performed only slightly above chance level. This is unsurprising, since Table 1 shows that there is only one instance where the mean vowel formant exhibited significant differences across load levels.

The trajectory offset parameters (LinRegIntercept, QuadRegOrd0) produced chance level classification performance in the 2-class results but seem to provide reasonably good performance in the 3 -class results. This might be because 


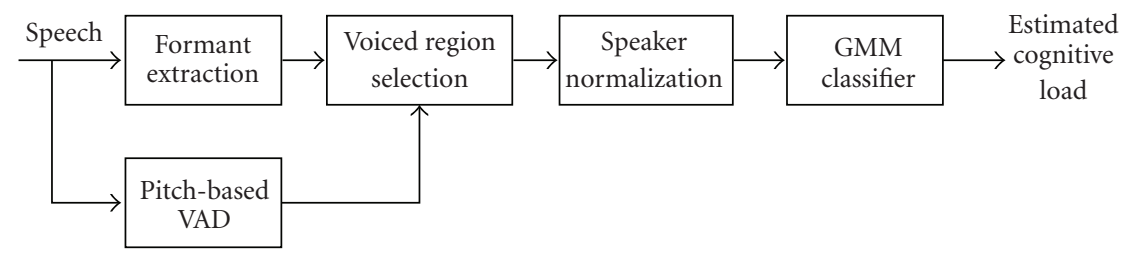

FIGURE 4: Block diagram of cognitive load classification system utilizing frame-based formant frequencies as front-end features.

the mean values of the formant frequencies are moving in different directions, for different load levels, as suggested by Figure 1. These vowel-specific shifts might not be accurately modeled, due to the limited amount of training data available, and the low number of Gaussian mixtures used. On the other hand, performance of the features was better in the 3 -class results, possibly because the features were affected by the artificially induced change in speech rate in the high-load task.

What is perhaps more surprising is the poor classification performance of StdDev, which do not agree with the statistical analysis of Table 1 . One possible reason for this inconsistency could be because the speaker normalization method used (speaker-specific feature warping) only preserves the rank order among feature frames but not their standard deviation. A similar reasoning can be used to explain the poor performance of Skewness as well.

In summary, the slope, minimum, range, and duration of the vowel formant trajectories seem to contain information useful for vowel-based cognitive load classification.

4.2. Utterance-Based Classification Using Frame-Based Formant Features. In this section, the performance of a cognitive load classification system using frame-based formant features is evaluated. The evaluation was performed on 2 separate databases: the Stroop test database and the readingcomprehension database.

4.2.1. Experimental Setup. Figure 4 shows the block diagram of the cognitive load classification system that was used in this section. Formant frequencies were automatically extracted from full utterances of both databases using the Wavesurfer/Snack toolkit, as described in Section 3.1. Formant frequencies for the reading-comprehension database were extracted using a slightly different configuration from the previous default configuration: the number of formants tracked was three instead of four and the sampling frequency used was $8 \mathrm{kHz}$ instead of $10 \mathrm{kHz}$. This was necessary because the default configuration resulted in significant proportion of erroneous formant tracks.

The formant frequencies were then normalized using speaker-specific feature warping, as explained in Section 4.1. The Praat software [27] was used as a pitch-based voice activity detector (VAD) to select voiced regions from the speech, and formant frequencies extracted from those voiced regions were used as features. Dynamic information was also considered through the use of regression-based delta coefficients. A regression window size of 9 frames was found to provide good classification performance.
TABle 4: Comparison of 2-class and 3-class classification results for different frame-based formant frequencies and MFCC features, evaluated on the Stroop test database.

\begin{tabular}{lcccc}
\hline Features & \multicolumn{2}{c}{ 2-class accuracy (\%) } & \multicolumn{2}{c}{ 3-class accuracy (\%) [14] } \\
& Without delta & With delta & Without delta & With delta \\
\hline MFCC & 70.4 & 80.6 & 53.3 & 65.2 \\
$\left\{F_{1}, F_{2}, F_{3}\right\}$ & 72.2 & 84.3 & 55.2 & 67.7 \\
$\left\{F_{1}, F_{2}\right\}$ & 71.3 & 75.0 & 55.8 & 65.2 \\
$\left\{F_{1}, F_{3}\right\}$ & 58.3 & 80.6 & 51.0 & 64.5 \\
$\left\{F_{2}, F_{3}\right\}$ & 63.9 & 69.4 & 44.7 & 60.3 \\
$F_{1}$ & 63.9 & 75.0 & 55.4 & 60.9 \\
$F_{2}$ & 69.4 & 77.8 & 53.4 & 58.9 \\
$F_{3}$ & 55.6 & 59.3 & 44.8 & 44.1 \\
\hline
\end{tabular}

The normalized formant frequencies of the voiced regions were then used as features to classify the cognitive load level of each utterance in the databases. A leaveone-speaker-out cross-validation evaluation was performed using a GMM/UBM classifier. A UBM with 32 Gaussian mixtures was trained using features extracted from a third database: the story reading database. MAP adaptation was then performed to adapt the means of the UBM using data from either the Stroop test or the reading-comprehension database.

4.2.2. Classification Results on the Stroop Test Database. Table 4 shows the 2-class (low and medium-load) and 3-class (low, medium, and high-load) classification results on the Stroop test database, with formant frequencies as features. The classification results are compared with MFCC features (7 MFCC, not including the zeroth coefficient), which are commonly used as a baseline in previous cognitive load classification systems $[9,13,28]$.

The first thing to notice is that the first three formant frequencies $\left\{F_{1}, F_{2}, F_{3}\right\}$ outperformed $M F C C$ in both the 2 -class and 3-class classification results. This is remarkable given that the formant features have lower dimensionality compared with MFCC (3 and 7, resp.), and the fact that the formant frequencies were not manually corrected in this experiment. Similar results can be observed when delta coefficients are considered. These results suggest that cognitive load information can be captured using lower dimensionality formant features. In general, incorporating delta coefficients into the feature sets resulted in an increase in classification performance. This agrees with the findings 
TABle 5: Comparison of 2-class and 3-class classification results for different frame-based formant frequencies and MFCC features, evaluated on the reading-comprehension database.

\begin{tabular}{lcccc}
\hline Features & \multicolumn{2}{c}{ 2-class accuracy (\%) } & \multicolumn{2}{c}{ 3-class accuracy (\%) } \\
& Without delta & With Delta & Without delta & With delta \\
\hline$M F C C$ & 63.3 & 71.1 & 48.9 & 52.6 \\
$\left\{F_{1}, F_{2}, F_{3}\right\}$ & 72.2 & 77.8 & 48.9 & 51.9 \\
$\left\{F_{1}, F_{2}\right\}$ & 56.7 & 61.1 & 32.6 & 39.3 \\
$\left\{F_{1}, F_{3}\right\}$ & 70.0 & 75.6 & 43.0 & 48.1 \\
$\left\{F_{2}, F_{3}\right\}$ & 65.6 & 71.1 & 39.3 & 45.9 \\
$F_{1}$ & 60.0 & 53.3 & 28.9 & 31.1 \\
$F_{2}$ & 52.2 & 64.4 & 36.3 & 45.9 \\
$F_{3}$ & 67.8 & 66.7 & 48.1 & 50.4 \\
\hline
\end{tabular}

of previous work $[9,19]$ that suggested that dynamic speech information is important for cognitive load classification.

In terms of the contribution of individual formants, results suggest that the first two formant frequencies provide majority of the cognitive load information. In the 2class classification results, performance of $F_{1}(63.9 \%)$ or $F_{2}(69.4 \%)$ was significantly better than the performance of $F_{3}(55.6 \%)$. In fact, the performance of $F_{2}$ alone was comparable with the performance of $\left\{F_{1}, F_{2}, F_{3}\right\} \quad(72.2 \%)$. A similar trend can be observed when delta coefficients are considered.

The trend is slightly different in the case of the 3-class classification results. Here, $F_{1}$ performs slightly better than $F_{2}$, whereas the opposite trend was observed in the 2-class classification results. This slight discrepancy might be due to the explicit change in speech rate that was induced in the high-load task. Nevertheless, the 3-class classification results do support the claim that $F_{1}$ and $F_{2}$ provide the greatest contribution in terms of cognitive load classification.

When score-level fusion, similar to [14], was applied to combine the $\left\{F_{1}, F_{2}, F_{3}\right\}$-based classification system with each of the statistical formant features system found in Section 4.1, no significant improvement in performance was attained. This suggests that most of the cognitive load information present in the statistical formant features is already captured by the frame-based formant features.

4.2.3. Classification Results on the Reading-Comprehension Database. To verify some of the claims made in the previous sections, we applied the same cognitive load classification system to a separate database.

Table 5 summarizes the classification results for different formant frequency-based features, evaluated on the readingcomprehension database. Once again, the classification performance of MFCC-based features was used as a baseline for comparison. Similarly to the Stroop test database, the 2-class classification results show $\left\{F_{1}, F_{2}, F_{3}\right\}$ outperforming MFCC in both static and dynamic cases (where delta coefficients were considered). In the case of the 3-class classification results, $\left\{F_{1}, F_{2}, F_{3}\right\}$ did not outperform $M F C C$, but its performance was still comparable: both $\left\{F_{1}, F_{2}, F_{3}\right\}$ and MFCC achieved the same classification accuracy of $48.9 \%$ in the static case, whereas in the dynamic case, the absolute difference between the classification accuracy of $\left\{F_{1}, F_{2}, F_{3}\right\}$ and MFCC was only $0.7 \%$. These results, once again, suggest that cognitive load information can be effectively captured using lower-dimensionality formant features.

Although the importance of dynamic features for cognitive load classification has been mentioned in $[9,19]$, the results have, until now, only been reported for the Stroop test database. The results here show that incorporating delta coefficients into the feature sets does improve cognitive load classification in general: performance improvements were observed for almost all feature sets in this database, except in the 2-class classification results of $F_{1}$ and $F_{3}$.

There are, however, some results here which do not agree with the results from the Stroop test database evaluation. In this evaluation, $F_{3}$ was found to perform better than $F_{1}$ or $F_{2}$. In fact, the 3-class classification results show that the performance of $F_{1}$ was below chance level, whereas the performance of $F_{2}$ was only slightly above chance level.

We believe that the differences in the results might be attributable to the different types of speech represented in the databases. As mentioned in Section 2, the Stroop test database consists of utterances which are made up of spoken color names. Speech corresponding to these color names is reasonably separated from one another temporally. Hence, the Stroop test database is almost like an isolated word database.

In contrast, the reading-comprehension database consists of continuous speech. This difference might affect the accuracy of the formant extraction algorithm. By visual inspection, we found the formant tracks of files in the Stroop test database to be smoother, and hence more reliable, than the formant tracks extracted from the readingcomprehension database. This also provides an explanation for the overall lower classification accuracy of features extracted from the reading-comprehension database when compared with the Stroop test database.

\section{Conclusion}

This work has shown that vowel formant frequencies can be correlated with cognitive load and can, hence, be used as effective features for cognitive load classification. In general, changes were observed, under different load conditions, for parameters related to the DC offset, the dispersion, and the slope of the formant trajectory. Analyses of formant parameters and formant trajectories have shown that changes in the formant trajectories seem to be stronger for certain vowel sounds. When the statistical formant parameters were used for vowel-based cognitive load classification, results show that cognitive load information is captured in the slope, range, and duration of the formant trajectory.

On the other hand, results from the utterance-based classification showed that the performance of frame-based formant features is comparable with, if not better than, frame-based MFCCs; this is despite formant features having a dimensionality of 3 compared with MFCCs with a dimensionality of 7. This finding was consistent across two different databases, hence suggesting that cognitive load 
information can be captured using features with lower dimensionality.

Apart from that, results from the frame-based classification and the earlier formant parameter analysis suggested that important cognitive load information is captured in the dynamic information of the formant frequencies. This was reflected by the improvement in the utterance-based classification performance when delta coefficients were included in the feature sets. Additionally, parameters that capture the temporal information of the formant trajectory (LinRegSlope and $D C T_{2}$ ) seem to exhibit significant differences, across different loads, for more vowels and formants; they also performed well in the vowel-based classification evaluation.

The strong trends exhibited by Duration in the statistical analysis and vowel-based classification motivate us to further investigate the effect of cognitive load on vowel and word duration. Future work includes investigating the duration of voiced regions as a potential feature for cognitive load classification.

\section{References}

[1] F. Paas, J. E. Tuovinen, H. Tabbers, and P. W. M. Van Gerven, "Cognitive load measurement as a means to advance cognitive load theory," Educational Psychologist, vol. 38, no. 1, pp. 63-71, 2003.

[2] F. G. W. C. Paas and J. J. G. Van Merriënboer, "Instructional control of cognitive load in the training of complex cognitive tasks," Educational Psychology Review, vol. 6, no. 4, pp. 351371, 1994.

[3] G. Camp, F. Paas, R. Rikers, and J. Van Merrienboer, "Dynamic problem selection in air traffic control training: a comparison between performance, mental effort and mental efficiency," Computers in Human Behavior, vol. 17, no. 5-6, pp. 575-595, 2001.

[4] M. Hoedemaeker and M. Neerincx, "Attuning in-car user interfaces to the momentary cognitive load," in Proceedings of the 3rd International Conference on Foundations of Augmented Cognition (FAC '07), vol. 4565 of Lecture Notes in Computer Science, pp. 286-293, 2007.

[5] M. St. John, D. A. Kobus, J. G. Morrison, and D. Schmorrow, "Overview of the DARPA augmented cognition technical integration experiment," International Journal of HumanComputer Interaction, vol. 17, no. 2, pp. 131-149, 2004.

[6] S. E. Lively, D. B. Pisoni, W. Van Summers, and R. H. Bernacki, "Effects of cognitive workload on speech production: acoustic analyses and perceptual consequences," Journal of the Acoustical Society of America, vol. 93, no. 5, pp. 2962-2973, 1993.

[7] A. Berthold and A. Jameson, "Interpreting symptoms of cognitive load in speech input," in Proceedings of the International Conference on User Modeling, pp. 235-244, 1999.

[8] C. Muller, B. Grossmann-Hutter, A. Jameson, R. Rummer, and F. Wittig, "Recognizing time pressure and cognitive load on the basis of speech: an experimental study," in Proceedings of the User Modeling, vol. 2109 of Lecture Notes in Computer Science, pp. 24-33, 2001.

[9] B. Yin, F. Chen, N. Ruiz, and E. Ambikairajah, "Speechbased cognitive load monitoring system," in Proceedings of the IEEE International Conference on Acoustics, Speech and Signal Processing (ICASSP '08), pp. 2041-2044, 2008.

[10] P. N. Le, E. Ambikairajah, E. H. C. Choi, and J. Epps, "A nonuniform subband approach to speech-based cognitive load classification," in Proceedings of the 7th International Conference on Information, Communications and Signal Processing (ICICS '09), pp. 1-5, 2009.

[11] T. F. Yap, J. Epps, E. H. C. Choi, and E. Ambikairajah, "Glottal features for speech-based cognitive load classification," in Proceedings of the IEEE International Conference on Acoustics, Speech and Signal Processing (ICASSP '10), pp. 5234-5237, 2010.

[12] P. Le, J. Epps, E. Choi, and E. Ambikairajah, "A study of voice source and vocal tract filter based features in cognitive load classification," in Proceedings of International Conference on Pattern Recognition, pp. 4516-4519, 2010.

[13] T. F. Yap, E. Ambikairajah, J. Epps, and E. H. C. Choi, "Cognitive load classification using formant features," in Proceedings of the 10th International Conference on Information Sciences, Signal Processing and Their Applications (ISSPA '10), pp. 221-224, 2010.

[14] T. F. Yap, J. Epps, E. Ambikairajah, and E. H. C. Choi, "An investigation of formant frequencies for cognitive load classification," in Proceedings of the 11th Annual Conference of the International Speech Communication Association (INTERSPEECH' 10), pp. 2022-2025, 2010.

[15] J. H. L. Hansen and S. Patil, "Speech under stress: analysis, modeling and recognition," in Proceedings of the Speaker Classification I, vol. 4343 of Lecture Notes in Computer Science, pp. 108-137, 2007.

[16] M. Goudbeek, J. P. Goldman, and K. R. Scherer, "Emotion dimensions and formant position," in Proceedings of the 10th Annual Conference of the International Speech Communication Association (INTERSPEECH '09), pp. 1575-1578, September 2009.

[17] C. Lee, S. Yildirim, M. Bulut et al., "Emotion recognition based on phoneme classes," in Proceedings of International Conference on Spoken Language Processing, pp. 889-892, 2004.

[18] B. Vlasenko, B. Schuller, A. Wendemuth, and G. Rigoll, "Frame vs. turn-level: emotion recognition from speech considering static and dynamic processing," in Proceedings of the 2nd International Conference on Affective Computing and Intelligent Interaction (ACII '07), vol. 4738 of Lecture Notes in Computer Science, pp. 139-147, 2007.

[19] B. Yin, N. Ruiz, F. Chen, and M. A. Khawaja, "Automatic cognitive load detection from speech features," in Proceedings of the Australasian Computer-Human Interaction Conference (OZCHI'07), pp. 249-255, 2007.

[20] MetaMetrics Inc., "The Lexile Framework® for Reading," 2010, http://www.lexile.com/.

[21] B. Lindblom, "Spectrographic study of vowel reduction," Journal of the Acoustical Society of America, vol. 35, no. 11, pp. 1773-1781, 1963.

[22] M. Pitermann, "Effect of speaking rate and contrastive stress on formant dynamics and vowel perception," Journal of the Acoustical Society of America, vol. 107, no. 6, pp. 3425-3437, 2000.

[23] J. S. Garofolo, L. F. Lamel, W. M. Fisher, J. G. Fiscus, D. S. Pallett, and N. L. Dahlgren, "DARPA TIMIT acousticphonetic continuous speech corpus CD-ROM," NTIS order number PB91-100354, 1993.

[24] K. Sjölander and J. Beskow, "Wavesurfer-an open source speech tool," in Proceedings of International Conference on Spoken Language Processing, vol. 4, pp. 464-467, 2000.

[25] R. A. Fox and E. Jacewicz, "Cross-dialectal variation in formant dynamics of American English vowels," Journal of the Acoustical Society of America, vol. 126, no. 5, pp. 2603-2618, 2009. 
[26] V. Sethu, E. Ambikairajah, and J. Epps, "Speaker normalisation for speech-based emotion detection," in Proceedings of the 15th International Conference on Digital Signal Processing (DSP '07), pp. 611-614, July 2007.

[27] P. Boersma and D. Weenink, "Praat: a system for doing phonetics by computer," 2008, http://www.praat.org/.

[28] T. F. Yap, E. Ambikairajah, E. Choi, and F. Chen, "Phase based features for cognitive load measurement system," in Proceedings of the IEEE International Conference on Acoustics, Speech, and Signal Processing (ICASSP '09), pp. 4825-4828, 2009. 